\title{
AVALIAÇÃO DA CONDIÇÃO BUCAL EM PACIENTES RENAIS CRÔNICOS SUBMETIDOS À HEMODIÁLISE
}

\author{
Cláudia Régia de Souza Dias, tatiana Cerveira Valois de Sá, antonio luiz amaral Pereira, Cláudia Maria Coelho alves* \\ Trabalho realizado no Departamento de Odontologia ll da Universidade Federal do Maranhão - UFMA, São Luís, MA
}

\author{
*Correspondência \\ Rua dos Sírius, 86 - Portal dos \\ Vinhais - São Luís - MA \\ Cep 65078-340 \\ Tel: (98) 3236-0529 \\ cacaucoelho@bol.com.br
}

\begin{abstract}
RESUMO
OBjetivos. Avaliar a condição bucal dos pacientes com insuficiência renal crônica submetidos à hemodiálise, no município de São Luís, comparando os três centros de referência da cidade e os tempos de hemodíalise.

Métodos. A amostra foi constituída por 107 pacientes, nos quais analisou-se o índice de placa (IP) e a prevalência de cárie dentária (CPO-D). Os pacientes foram categorizados em três grupos, de acordo com o tempo de hemodiálise (3 meses a $<$ I ano, I a 3 anos e $>3$ anos). Os dados obtidos foram submetidos aos testes estatísticos ANOVA e Kruskal-Wallis, com nível de significância de $5 \%$.

Resultados. As médias/desvios padrão do IP nos grupos foram, respectivamente, $0.91 \pm 0.61 ; 1.04 \pm 0.60$ e $1.25 \pm 0.67$. Quanto ao CPO-D destes grupos, as médias/desvios padrão foram 13.63 \pm 8.91 ; $13.89 \pm 8.12$ e $16.79 \pm 7.31$. Não houve diferenças significativas entre os centros, nem entre os tempos de hemodiálise estudados.

Conclusäo.O tempo de tratamento da doença não alterou ou interferiu no acúmulo de placa bacteriana e na prevalência de cárie dentária. Houve uniformidade na condição bucal dos pacientes nos três centros estudados.

Unitermos: Insuficiência renal crônica. Saúde bucal. Cárie dentária. Epidemiologia.
\end{abstract}

\section{INTRODUÇÃO}

A insuficiência renal crônica (IRC) é uma doença sistêmica, relativamente comum, que implica na redução ou limitação da capacidade de filtração glomerular dos rins, causando a uremia, que se caracteriza pelo acúmulo no sangue de substâncias que deveriam ser filtradas e excretadas pelos rins, ocorrendo o comprometimento deste órgão e levando o paciente a fazer uso da hemodiálise.

A IRC pode ocasionar determinadas mudanças sistêmicas, tais como alterações cardiovasculares (hipertensão arterial, aterosclerose, pericardites, cardiomiopatias, arritmias cardíacas e hipertensão pulmonar), anemia, problemas hemostáticos e linfocitopenia'. Também podem ser observadas alterações ósseas e bioquímicas, além de alterações gastrointestinais e dermatológicas ${ }^{2}$.

Tais mudanças sistêmicas podem refletir no meio bucal, levando a alterações na composição salivar, com aumento dos níveis de uréia, potássio, fosfato ${ }^{3,4,5,6}$ e diminuiçãa nos de $\mathrm{Ca}$ e $\mathrm{Mg}^{7}$; no nível do fluxo salivar, com diminuiçã̃o do mesmo, ${ }^{4,8-15}$; no pH salivar, que pode ser mais alcalino ${ }^{6}$; na capacidade-tampão, com aumento da mesma $a^{10,14,16}$ e elevada formação de cálculo dentário ${ }^{17}$.

Além disso, a falta de cuidados com a higiene bucal pode afetar a saúde bucal destes pacientes, resultando em mais cáries, doenças periodontais e lesões bucais ${ }^{4,15,18}$. Não há consenso quanto à predisposição destes pacientes à cárie dentária. Em pesquisa realizada por Bayraktar et al. ${ }^{14}$, foi observado que não houve mudança quanto à ocorrência de cárie dentária. Já no estudo de Naugle et al. ${ }^{19}$, observou-se que a experiência de cárie dentária foi elevada nos pacientes em diálise, enquanto que nas pesquisas realizadas por AlNowaiser et al. ${ }^{16}$, Jaffe et a $1 .{ }^{20}$ e Davidovich et al. ${ }^{21}$, pacientes com IRC em diálise apresentaram menores índices de cárie dentária.

Com relação à presença de placa bacteriana, alguns estudos 9, 16,21${ }^{24}$ têm demonstrado que pacientes com IRC apresentam maior acúmulo da mesma e, devido a alta concentração de uréia presente na saliva destes pacientes, possuem grande quantidade de cálculo dentário, assim como tendência de hálito urêmico ${ }^{4}, 9$

Considerando que não há consenso quanto à predisposição destes pacientes ao desenvolvimento da cárie dentária, e que a presença de placa sem o efetivo controle pode levar à cárie dentária, justifica-se a realização deste estudo, que teve como objetivo avaliar a prevalência de cárie dentária e o acúmulo de placa bacteriana nos pacientes com insuficiência renal crônica submetidos à hemodiálise.

\section{Métodos}

Trata-se de um estudo transversal em que foi avaliada a prevalência de cárie dentária e a presença de placa bacteriana em pacientes com insuficiência renal crônica (IRC) submetidos à hemodiálise, no município de São Luís. Comparou-se os pacientes que freqüentavam os Centros de Referência da cidade (Pró-Renal, Hospital Universitário Presidente Dutra da Universidade Federal do Maranhão - HUUFMA e Centro de Nefrologia e Hipertensão Arterial - CENEFRON) e os tempos de tratamento em hemodiálise ( 3 meses a $<$ de $\mid$ ano, I a 3 anos e $>$ de 3 anos). 


\begin{tabular}{|c|c|c|}
\hline \multicolumn{3}{|c|}{$\begin{array}{c}\text { Tabela I-Dados descritivos dos pacientes quanto à idade, ao sexo, } \\
\text { à escolaridade eà renda mensal própria }\end{array}$} \\
\hline \multirow[b]{2}{*}{ Variável } & \multicolumn{2}{|c|}{$\underline{\operatorname{IRC}(n=107)}$} \\
\hline & $\mathrm{n}$ & $\%$ \\
\hline \multicolumn{3}{|l|}{ Idade } \\
\hline $20-29$ & 22 & 20.56 \\
\hline $30-39$ & 19 & 17.76 \\
\hline $40-49$ & 28 & 26.17 \\
\hline $50-59$ & 21 & 19.62 \\
\hline $60-69$ & 13 & 1215 \\
\hline $70-79$ & 2 & 1.87 \\
\hline $80-89$ & 2 & 1.87 \\
\hline \multicolumn{3}{|l|}{ Sexo } \\
\hline M & 48 & 44.86 \\
\hline $\mathrm{F}$ & 59 & 55.14 \\
\hline \multicolumn{3}{|l|}{ Escolaridade } \\
\hline Analfabeto & 9 & 8.41 \\
\hline $1^{\circ}$ grau incompleto & 57 & 53.27 \\
\hline $1^{\circ}$ grau completo & 8 & 7.47 \\
\hline $2^{\circ}$ grau incompleto & 4 & 3.74 \\
\hline $2^{\circ}$ grau completo & 23 & 21.50 \\
\hline $3^{\circ}$ grau incompleto & 3 & 2.80 \\
\hline $3^{\circ}$ grau completo & 3 & 2.80 \\
\hline \multicolumn{3}{|l|}{ Renda } \\
\hline Ausência & 10 & 9.34 \\
\hline < | salário & 13 & 1215 \\
\hline | salário & 59 & 55.14 \\
\hline > I a 3 salários & 19 & 17.76 \\
\hline > 3 salários & 6 & 5.61 \\
\hline
\end{tabular}

Este estudo foi aprovado pelo Comitê de Ética em Pesquisa do Hospital Universitário Presidente Dutra da Universidade Federal do Maranhão - HUUFMA (Parecer n 006/2005). Todos os pacientes participantes foram informados do caráter da pesquisa e assinaram um "Termo de Consentimento Livre e Esclarecido", com autorizaçãa, por escrito, de sua participação no estudo. A pesquisa foi iniciada mediante autorização dos diretores clínicos dos centros de hemodiálise.

A população de pacientes com IRC submetidos a hemodiálise (três sessões semanais), no município de São Luís, era de 453, distribuídos no três Centros de Referência, sendo que II 5 pertenciam ao Pró-Renal, 262 ao CENEFRON e 76 ao Hospital Universitário Presidente Dutra. A amostra deste estudo foi composta por 107 pacientes, de ambos os sexos, com idades entre 20 e 87 anos, selecionados aleatoriamente. Estes foram categorizados em três grupos, de acordo com o tempo em que faziam a hemodiálise, assim distribuídos: entre 3 meses e $<$ I ano, de I a 3 anos e acima de três anos. $O$ estudo foi realizado no período de junho a outubro de 2006.

Foram excluídos os pacientes edêntulos, aqueles com tratamento iniciado a menos de três meses, e portadores de outras doenças sistêmicas, tais como diabetes, lúpus eritematoso e amiloidose. Antes de serem examinados, os pacientes seleciona- dos foram submetidos a um questionário/ entrevista, no qual foram registrados seus dados, como nome completo, endereço, sexo e idade, além de sua condição sistêmica, hábitos de higiene bucal e tempo de duração da doença.

A prevalência de cárie foi identificada por meio do índice CPOD (dentes cariados, perdidos e obturados), preconizado pela $\mathrm{OMS}^{25}$, enquanto que a presença ou não de placa bacteriana foi observada pelo índice de placa $(I P)^{26}$, em que foram avaliadas as quatro superfícies lisas do dente, sendo que cada uma recebeu escore de 0 a 3. Os escores foram somados e divididos por quatro, obtendo-se o índice de placa por dente. Ao somar os índices dos dentes e dividi-los pelo número de dentes examinados, o índice de placa para o paciente foi obtido de acordo com os seguintes critérios: 0 - ausência de placa na região gengival, I - leve acúmulo de placa aderida à gengiva marginal livre e áreas adjacentes do dente. A placa pode ser vista apenas após a aplicação de solução evidenciadora, ou por meio do uso da sonda na superfície dentária, 2 - acúmulo moderado de depósitos dentro da bolsa gengival ou no dente e margem gengival que pode ser vista ao exame clínico e 3 - abundância de placa dentro do sulco gengival e/ou no dente e margem gengival. O exame foi feito com o auxílio da sonda WHO-62I e do espelho bucal, sob luz artificial e na própria cadeira de hemodiálise. Os exames foram feitos somente por um examinador, sendo que os dados foram anotados na ficha por um auxiliar.

A análise estatística foi feita utilizando-se o programa Bioestat 4.0. Os dados obtidos foram submetidos aos testes de Shapiro Wilk e Lilliefors, para avaliar quanto à normalidade da distribuição dos dados. Os testes estatísticos de análise de variância (ANOVA) e Kruskal-Wallis foram usados para verificar a existência ou não de diferenças significativas acerca dos índices entre os centros de hemodiálise e os tempos de hemodiálise. O teste de Kruskal-Wallis foi aplicado para a avaliação do IP, devido o mesmo ser uma variável categórica, e para os casos em que o CPO-D e seus componentes não tiveram distribuição normal $(p<0.05)$. A análise de variância (ANOVA) foi usada quando os dados da variável apresentaram distribuição normal $(p>0.05)$. O nível de significância utilizado em todos os testes foi de $5 \%$.

\section{Resultados}

A Tabela I possui os dados descritivos da amostra estudada. Na análise dos dados obtidos, foi observado que a amostra ficou assim constituída: 48 indivíduos do sexo feminino (44,9\%) e 59 do sexo masculino (55, I\%). A média de idade dos pacientes foi de 44,64 \pm 14,89 anos, e o tempo médio em hemodiálise do grupo foi de 3,2 $\pm 2,28$ anos. As médias/desvios padrão do IP, CPO-D, cariado $(C)$, perdido $(P)$ e obturado $(O)$ nos pacientes estudados foram, respectivamente: $0,64 \pm 0,73 ; \quad \mid 4,77 \pm 8,03 ; \quad 2,95 \pm 2,70$; $\mid 1,42 \pm 8,69$ e $0,47 \pm 1,51$.

A estatística descritiva, assim como a análise de variância (ANOVA) e o teste Kruskal-Wallis para o IP e CPO-D nos centros e grupos encontram-se, respectivamente, nas Tabelas 2 e 3.

A análise de variância e o teste de Kruskal-Wallis não revelaram diferenças estatisticamente significativas acerca dos centros e dos 


\begin{tabular}{|c|c|c|c|c|c|c|}
\hline \multicolumn{7}{|c|}{ Tabela 2-IP, CPO-D, C, Pe O dos pacientes nos três centros de hemodiálise de São Luís-MA } \\
\hline Centro & $\mathbf{N}$ & $\mathbf{I P}$ & CPO-D & C & $\mathbf{P}$ & 0 \\
\hline HUUFMA & 35 & $0.87 \pm 0.47$ & $13.6 \pm 7.62$ & $2.8 \pm 2.31$ & $10.63 \pm 8.1$ & $0.17 \pm 1.01$ \\
\hline CENEFRON & 41 & $1.12 \pm 0.57$ & $15.66 \pm 8.13$ & $3.22 \pm 3.22$ & $11.83 \pm 9.15$ & $0.80 \pm 1.95$ \\
\hline \multirow[t]{3}{*}{ Pró-Renal } & 31 & $1.29 \pm 0.81$ & $14.94 \pm 8.47$ & $2.77 \pm 2.4$ & $11.77 \pm 8.94$ & $0.39 \pm 1.28$ \\
\hline & & $H^{*}=3.9867$ & $F * * * * 6.6232$ & $H^{*}=0.0598$ & $H^{*}=0.3745$ & $H^{*}=1.1533$ \\
\hline & & $p=0.1362$ & $p=0.5431$ & $p=0.9705$ & $p=0.8293$ & $p=0.5618$ \\
\hline \multicolumn{7}{|l|}{$\begin{array}{l}\text { *Kruskal-Wallis } \\
\text { **ANOVA } \\
a ́=0.05\end{array}$} \\
\hline \multicolumn{7}{|c|}{ Tabela 3 - IP, CPO-D, C, P e O dos pacientes em relação aos tempos de hemodiálise } \\
\hline $\begin{array}{l}\text { Tempode } \\
\text { Hemodiálise }\end{array}$ & $\mathbf{N}$ & IP & CPO-D & $\mathrm{C}$ & $\mathbf{P}$ & 0 \\
\hline $\begin{array}{c}3 \text { meses a } \\
<\text { | ano } \\
\text { | a } 3 \text { anos } \\
>3 \text { anos }\end{array}$ & $\begin{array}{l}16 \\
57 \\
34\end{array}$ & $\begin{array}{l}0.91 \pm 0.61 \\
1.04 \pm 0.60 \\
1.25 \pm 0.67 \\
H^{*}=1.7652 \\
p=0.4137\end{array}$ & $\begin{array}{l}|3.63 \pm 8.9| \\
\mid 3.89 \pm 8.12 \\
|6.79 \pm 7.3| \\
H^{*}=2.9278 \\
p=0.23 \mid 3\end{array}$ & $\begin{array}{c}2.87 \pm 2.22 \\
2.70 \pm 2.41 \\
3.41 \pm 3.32 \\
H^{*}=0.6319 \\
p=0.7291\end{array}$ & $\begin{array}{l}10.75 \pm 9.74 \\
10.98 \pm 8.68 \\
12.47 \pm 8.37 \\
H^{*}=1.2716 \\
p=0.5295\end{array}$ & $\begin{array}{c}0.00 \pm 0.00 \\
0.35 \pm 1.20 \\
0.91 \pm 2.15 \\
H^{*}=1.0994 \\
p=0.5771\end{array}$ \\
\hline
\end{tabular}

*Kruskal-Wallis

á $=0.05$

tempos de hemodiálise para ambos os índices, assim como nos componentes do CPO-D.

\section{Discussão}

De acordo com os resultados desta pesquisa, observou-se que a média/desvio padrão do IP dos pacientes foi de 0,64 $\pm 0,73$. Baseado na metodologia de Silness; Löe ${ }^{26}$, os pacientes com IRC apresentaram um leve acúmulo de placa bacteriana. Resultados semelhantes foram achados por Al - Wahadni; Al Omari ${ }^{27}$, Atassi ${ }^{18}$, Naugle et al. ${ }^{19}$, Davidovich et al.. ${ }^{21}$, Klassen; Krasko ${ }^{24}$, Gavaldá et al. ${ }^{9}$ e Wolff et al.22. É provável que este acúmulo de placa bacteriana tenha ocorrido não como consequêencia direta da doença, mas sim como reflexo indireto da mesma, já que, possivelmente, os pacientes se preocupam ou se dedicam tanto à doença que acabam negligenciando os cuidados de higiene bucal.

A amostra examinada apresentou um elevado índice CPO-D $(\mid 4,77 \pm 8,03)$, evidenciando tanto a necessidade de tratamento/ reabilitação como de medidas preventivas. Nos trabalhos realizados por Al Wahadni; Al Omari27, Gavaldá et al. ${ }^{9}$, Naugle et al. ${ }^{19}$, Fujimaki; Rosa; Torres ${ }^{8}$, Bayraktar et al..$^{14}$ e Bots et al. ${ }^{28}$, foram observados resultados semelhantes. Pode-se aferir que esses resultados também são conseqüências indiretas da doença, assim como da condição socioeconômica, em que 53,27\% (57) destes pacientes têm somente $1^{\circ}$ grau incompleto e $55,14 \%$ (59) têm renda mensal própria de apenas um salário mínimo. A maioria destes pacientes $(77,57 \%)$ busca atendimento odontológico somente em casos de dor/exodontia, negligenciando o atendimento preventivo. Isto faz com que tais pacientes apresentem níveis elevados de dentes perdidos e baixos níveis de dentes obturados.

Quando se comparou os três centros de hemodiálise em relaçãa a ambos os índices, foi observada uma uniformidade nas condições dos pacientes. Isto pode sugerir que a maioria destes pacientes apresenta semelhantes condições socioeconômicas, hábitos de higiene bucal e atendimento odontológico, como observado em nossos resultados. Em relaçãa aos hábitos de higiene bucal, Atassi ${ }^{18}$ observou, em estudo realizado comparando pacientes com IRC em relação ao tempo de hemodiálise $(<\mid$ ano, I a 3 anos e $>$ de 3 anos), que não houve diferenças significativas acerca dos três grupos em relação à freqüência de escovação.

Pode ser observada também uma tendência de aumento dos índices estudados com a maior duração do tempo de diálise. Isto pode refletir as preocupações que tais pacientes têm com sua condição sistêmica, negligenciando os cuidados com a higiene bucal. Quanto à comparação, em relação aos tempos de hemodiálise para ambos os índices, observou-se que não houve diferenças estatisticamente significativas. Em relação ao IP, estes achados estão de acordo com os de Naugle et al. ${ }^{19}$ e Al Wahadni; Al Omari ${ }^{27}$, e diferem dos resultados encontrados por Atassi ${ }^{18}$ e Davidovich et al. ${ }^{21}$, em que o primeiro observou diferenças estatisticamente significativas entre os pacientes submetidos à 
hemodiálise há menos de I ano e os de I a 3 anos, assim como entre os de menos de I ano e os acima de 3 anos. Já Davidovich et al. ${ }^{21}$ estudaram a correlação entre parâmetros bucais e duração da IRC nos pacientes em diálise, observando que a duração da diálise estava correlacionada com o aumento do índice de placa.

Em relação ao CPO-D, os achados deste estudo são semelhantes àqueles encontrados por Naugle et al. ${ }^{19}$, no entanto diferem dos de Al- Wahadni; Al- Omari ${ }^{27}$, que observaram diferenças estatisticamente significativas no CPO-D e no componente cariado entre os pacientes com menos de I ano e de I a 3 anos de hemodiálise, assim como nos de menos de I ano e os com mais de 3 anos. 0 objetivo da divisão dos pacientes de acordo com o tempo de hemodiálise foi avaliar o efeito da duração da doença na saúde bucal dos mesmos. Esta divisão foi baseada em estudos similares (Atassi ${ }^{18}$, Naugle et al. ${ }^{19}$ e Al- Wahadni; Al- Omari ${ }^{27}$ ).

Um fato que deve ser considerado neste estudo é a grande diferença de anos existente entre os pacientes em hemodiálise há mais de 3 anos e aqueles em hemodiálise há 3 meses a $<1$ ano e I a 3 anos, sendo que metodologias diferentes, principalmente aquelas que permitam um acompanhamento das condições bucais dos pacientes durante toda duração da doença, podem evidenciar resultados diferentes em relação às variáveis estudadas.

Os resultados apresentados mostram elevado CPO-D e número de dentes perdidos nos pacientes com IRC. Isto sugere a necessidade da instalação de um programa preventivo/ terapêutico bucal direcionado a este grupo de pacientes, assim como de um acompanhamento regular destes, objetivando conscientizá-los da importância da saúde bucal, principalmente devido à sua condição sistêmica, uma vez que muitos necessitam de um grau aceitável de saúde bucal para a realização/manutenção de um possível transplante renal. Necessita-se de estudos comparativos e/ou de associação que respaldem os resultados encontrados, assim como direcionem aos fatores que levam às alterações bucais em tais pacientes.

\section{Conclusão}

Pode-se concluir que os pacientes avaliados apresentaram elevada média de CPO-D e número de dentes perdidos $(P)$, baixo nível de dentes obturados, além de índice de placa (IP) de leve a moderado. O tempo de tratamento da doença não alterou ou interferiu no acúmulo de placa bacteriana e na prevalência de cárie dentária. A condição bucal dos pacientes nos três centros de hemodiálise estudados foi semelhante.

\section{Conflito de interesse: não há.}

\section{SUMMARY}

EVALUATION OF ORAL CONDITION OF PATIENTS WITH CHRONIC RENAL FAILURE SUBMITTED TO HEMODIALYSIS

BACKGROUND. Evaluate the oral conditions of patients with chronic renal failure submitted to hemodialysis in the city of São Luís, by comparing three reference centers and the times of hemodialysis.
Methods. The sample consisted of 107 patients, distributed among the centers. Plaque Index (IP) and Dental Caries Prevalence (DMF-T) were evaluated. Patients were subdivided into three groups according to time of hemodialysis (3 months to $<1$ year, $I$ the 3 years and $>3$ years). Data were analyzed using the ANOVA and the Kruskal-Wallis test with a level of significance of $5 \%$.

RESULTS. Plaque index averages in the groups were respectively, $0.91 \pm 0.67 ; 1.04 \pm 0.60 ; 1.25 \pm 0.61$. The averages of DMF-T in the respective groups were 13.63 $\pm 8.91 ; 13.89 \pm 8.12$; 16.79 \pm 7.31 . There were no significant differences between the centers of reference and the times of hemodialysis.

CONCLUSION. Time of treatment of the disease did not change or interfere on plaque accumulation and prevalence of the dental caries. There was uniformity in the patients' oral condition in the three reference centers under study. [Rev Assoc Med Bras 2007; 53(6): $5 \mid 0-4]$

KEY WORDS: Renal insufficiency. Oral health. Dental caries. Epidemiology.

\section{REFERÊNCIAS}

I. Stein A, Wild J. Kidney failure explained. 2nd ed. London: Class Publishing, 2002, p.5.

2. Fraser R, Cosmo L. Primer on kidney diseases. S Diego: Academic Press, 1994

3. Blum M, Zurkowsky S, Gilad J, Aviram A. Salivary phosphate and calcium concentrations in uremia. Clin Nephrol. 1970; |2(4): |9|-2.

4. Epstein SR, Mandell, Scoopp IW. Salivary composition and calculus formation in patients undergoing hemodialysis. I Periodontol. 1980;5।(6):336-8.

5. Shasha SM, Ben Aryeh H, Angel A, Gutman D. Salivary content in hemodialysed patients. J Oral Med. 1983; 38(2):67-70.

6. Peterson S, Woodhead J, Crall, J. Caries resistence in children with chronic renal failure: plaque $\mathrm{pH}$, salivary $\mathrm{pH}$, and salivary composition. Pediatr Res. 1985;19(8):796-9.

7. Obry F, Belcourt AB, Frank RM, Fischbach M. Biochemical study of whole saliva from children with chronic renal failure. ASDC J Dent Chile. 1987;54(6)429-32.

8. Fujimaki M, Rosa OPS, Torres AS. Microrganismos cariogênicos em pacientes com insuficiência renal crônica em hemodiálise. Rev Odontol Univ São Paulo. 1998;12(2):|49-58.

9. Gavaldá C, Bagan J, Scully C, Silvestre FJ, Milián MA, Jiménez Y. Renal hemodialysis patients: oral, salivary, dental and periodontal findings in 105 adult cases. Clin Oral Med. 1999;5(4):299-302.

10. Kho HS, Lee SW, Cheng SC, Kim YK. Oral manifestations and salivary flow rate, $\mathrm{pH}$, and buffer capacity in patients with end-stage renal disease undergoing hemodialysis. Oral Surg Oral Med Oral Pathol Oral Radiol End. 1999;88(1):316-9.

I I. Kao CH, Hsieh JF, Tsai SC, Ho YJ Chang HR. Decreased salivary fuction in patients with end-stage renal disease requiring hemodialysis. Am J Kidney Dis. 2000;36(6): 1110-4.

12. Kaya M, Çermik TF, Üstun F, Sen S, Bernarda S. Salivary fuction in patients with chronic renal failure undergoing hemodialysis. Ann Nucl Med. 2002; I6(2): I 17-20.

13. Postorino M, Catalano C, Martorano C, Cutrupi S, Marino C, Cozzupoli P, Scudo P, et al. C. Salivary and lacrimal secretion is reduced in patients with ESRD. Am J Kidney Dis. 2003;42(4):722-8.

I 4. Bayraktar G, Kazancioglu R, Bozfakioglu S, Yildiz A, Ark E. Evaluation 
of salivary parameters and dental status in adult hemodialysis patients. Clin Nephrol. 2004;62(5):380-3.

15. Proctor R, Kumar N, Stein A, Moles D, Porter S. Oral e dental aspects of chronic renal failure. J Dent Res. 2005;84(3):199-208.

16. Al-Nowaiser A, Roberts GJ, Trompeter RS, Wilson M, Lucas VS. Oral health in children with chronic renal failure. Pediatr Nephrol. 2002; 1 8( I):39-45.

17. Dias CRS, Libério SA, Guerra RNM, Monteiro S, Silveira EJD, Pereira ALA. Avaliação da condição periodontal de pacientes renais em hemodiálise. Rev Assoc Med Bras. 2005;5I(5):285-9.

18. Atassi F. Oral home care and the reasons for seeking dental care by individuals onrenal dialysis. J Contempy Dent Pract. 2002;3(2):3 I-4I.

19. Naugle K, Darby ML, Barman DB, Lineberger T, Powers R. The oral health status of individuals on renal dialysis. Ann Periodontol. 1998;3(1): 197-205.

20. Jaffe EC, Roberts GJ. Dental findings in chronic renal failure. Br Dent J. 1986;160(1):18-20.

2I. Davidovich E, Schwarz Z, Davidovitch M, Eidelman E, Bimstein E. Oral findings and periodontal status in children, adolescents and young adults suffering from renal failure. J Clin Periodontol. 2005;32( I0): I076-89.

22. Wolff A, Stark H, Sarnat H, Binderman I, Eisenstein B, Drukker A. The dental status of children with chronic renal failure. J Intr Pediatr Nephrol. 1985;6(2): 1 27-32.
23. Rahman MM, Çaglayan F, Rahman B. Periodontal health parameters in patients with renal failure and renal transplants receiving immunosuppressive therapy. J Nih Univ Sch Dent. 1992;34(4):26572.

24. Klassen JT, Krasko, BM. The dental health status of dialysis patients. J Can Dent Assoc. 2002;68(I):34-8.

25. World Health Organization WHO. Oral health survey: basic methods. $4^{\text {th }}$ ed. Geneva: ORH/EPID; 1997.

26. Silness J, Löe H. Periodontal disease in pregnancy II. Correlation between oral hygiene and periodontal condition. Acta Odontol Scand. 1964;22:121-35.

27. Al-Wahadni A, Al- Omari MA. Dental diseases in a jordanian population on renal dialysis. Quintessence Int. 2003;34:343-7.

28. Bots CP, Pooeterman JHG, Brand HS, Kalsbeek H, Van Amerongen $\mathrm{BM}$, Veerman $\mathrm{ECl}$, Nieuw Amerongen AV. The oral health status of dentate patients with chronic renal failure undergoing dialysis therapy. Oral Dis. 2006;12:176-80

Artigo recebido: 20/04/07

Aceito para publicação: I |/08/07 